\title{
Evaluación por acelerometría del ejercicio realizado por escolares chilenos durante una clase de educación física y su relación con el estado ponderal Accelerometer-based assessment of exercise in Chilean schoolchildren during a physical education class and its relationship with ponderal mass \\ *Marcelo Tuesta, **Fernando Barraza-Gómez, ***Alfredo Polgatiz Gajardo, ${ }^{* * * *}$ Rodrigo Yáñez-Sepúlveda, *****Eduardo Báez- San Martín
}

*Universidad Andres Bello (Chile), **Universidad Viña del Mar (Chile), ***Universidad Mayor (Chile), ****Pontificia Universidad Católica de Valparaíso (Chile), *****Universidad de Playa Ancha

Resumen. Introducción: La clase de educación física (CEF) debería ser capaz de activar las recomendaciones de ejercicio necesario para para promover un estilo de vida saludable en los niños. Objetivo: Comparar la actividad metabólica, gasto energético, cantidad de ejercicio e intensidad de esfuerzo físico realizado durante una CEF en niños en relación a su estado ponderal. Participantes y Métodos: Un total de 63 niños (8.0 \pm 1.4 años) y 57 niñas escolares ( $8.3 \pm 1.6$ años) fueron distribuidos en los grupos normopeso (NP), sobrepeso (SP) y obesos (OB) según su índice de masa corporal, y analizados sus desplazamientos corporales con acelerómetros durante una CEF. Resultados: El gasto energético relativo al peso corporal inducido por el ejercicio fue mayor en el grupo NP ( $4.7 \pm 1.8 \mathrm{kcal} / \mathrm{kg})$ respecto al SP $(3.4 \pm 1.7 \mathrm{kcal} / \mathrm{kg})$ y OB $(2.8 \pm 1.3 \mathrm{kcal} / \mathrm{kg})$ con $\mathrm{P}<0.05$. Asimismo, el grupo NP se mantuvo más tiempo de la CEF en intensidad de ejercicio muy vigoroso ( $2.3 \pm 2.2 \mathrm{~min})$ y menos en ejercicio ligero $(9.5 \pm 2.6 \mathrm{~min})$ que el grupo $\mathrm{OB}(1.8 \pm 2.5 \mathrm{~min} \mathrm{y} 11.1 \pm 2,3 \mathrm{~min}$, respectivamente) con $\mathrm{P}<0.05$. No hubo diferencias en la cantidad de movimiento (número de pasos) y actividad metabólica (MET) entre los grupos ( $\mathrm{P}>0.05)$. Conclusión: Los escolares NP son capaces de gastar más energía en relación a su peso corporal y con una mayor intensidad de ejercicio que sus pares OB durante una CEF.

Palabras claves: Gasto energético, Educación Física, Acelerometría.

Abstract. Background: The physical education subject (PES) should be capable of activating the exercise recommendations required to promote healthy lifestyle in children. Objective: To compare metabolic activity, energy expenditure, amount of exercise, and intensity of physical effort in children during a PES class, and to relate them to their body mass index. Participants and Methods: A total of 120 schoolchildren (63 boys aged $8.0 \pm 1.4$ years old; 57 girls aged $8.3 \pm 1.6$ years old) were grouped in normal weight (NW), overweight $(\mathrm{OW})$, and obese (OB) categories based on their body mass index, and their body movements were measured with accelerometer during a PES class. Results: Energy expenditure related to body weight induced by exercise was higher in the NW group ( $4.7 \pm 1.8 \mathrm{kcal} / \mathrm{kg})$ than in the $\mathrm{OW}(3.4 \pm 1.7 \mathrm{kcal} / \mathrm{kg})$ and $\mathrm{OB}(2.8 \pm 1.3 \mathrm{kcal} / \mathrm{kg})$ groups, with $\mathrm{P}<0.05$. Additionally, the $\mathrm{NW}$ group remained at very vigorous effort levels longer during the PES class $(2.3 \pm 2.2 \mathrm{~min})$, as well as they spent less time on light exercise $(9.5 \pm 2.6 \mathrm{~min})$ than the OB group $(1.8 \pm 2.5 \mathrm{~min}$ and $11.1 \pm 2.3 \mathrm{~min}$, respectively), with $\mathrm{P}<0.05$. There were no differences in the number of movements (total steps) and metabolic activity (MET) between groups $(\mathrm{P}>0.05)$. Conclusions: NW schoolchildren are able to spend more energy in relation to their body weight and with a greater intensity of exercise than their OB peers during a PES class.

Keywords: Physical education, accelerometry, body mass index.

\section{Introducción}

La obesidad es uno de los factores de riesgo que contribuye mayormente en la adquisición de enfermedades crónicas no transmisibles (ECNT) tales como hipertensión, diabetes y/o síndrome metabólico (OMS, 2012). Es reconocido que la práctica regular de ejercicio físico y el control de la alimentación son necesarios para mantener un adecuado equilibrio del peso corporal (Celis-Morales et al., 2015; Cuadri Fernandez, Tornero Quinones, Sierra Robles, \& Saez Padilla, 2018). Para controlar el peso corporal en niños, la Organización Mundial de la Salud recomienda realizar ejercicio al menos 60 minutos diarios a una intensidad moderada-vigorosa, o caminar 12000 pasos diarios (OMS, 2010a, 2010b). Esta actividad debería provocar un gasto energético mayor o igual a 1000 kcal semanales (Blair, 2009; Sesso, Paffenbarger, \& Lee, 2000). El cumplimiento de estas recomendaciones permitirá disminuir la probabilidad de padecer obesidad durante la adultez (Singh, Mulder, Twisk, van Mechelen, \& Chinapaw, 2008), evitando así, el desarrollo de ECNT(Blair, 2009; Troiano

Fecha recepción: 10-09-18. Fecha de aceptación: 05-08-19 Marcelo Tuesta-Roa

marcelotuesta@gmail.com et al., 2008). Si bien, la presencia de obesidad puede estar determinada por un factor genético (Bouchard, 1991), su prevalencia se encuentra relacionada con el desequilibrio energético positivo, es decir con el aumento del consumo de dietas con elevado contenido calórico y una disminución o falta de actividad física regular (Jebb \& Prentice, 1995). Un aspecto clave en el desarrollo de obesidad durante la infancia es el aumento del sedentarismo (Meneses Montero \& Ruiz Juan, 2017; Pasic et al., 2014).

La Organización para la Cooperación y Desarrollo Económico (OCDE), en su publicación «Obesity Update» del año 2014, ubicó a Chile entre los países con las tasas más altas de sobrepeso (30\%) y obesidad (25\%) infantil en mayores de seis años (OECD, 2014). Este panorama resulta aún más desalentador en edades más avanzadas, ya que en la última encuesta nacional de salud (ENS) realizada en nuestro país, las prevalencias de sobrepeso y obesidad para los jóvenes mayores de 14 años fueron 39.3\% y 25.1\% respectivamente (MINSAL, 2003, 2010a, 2010b). Uno de los objetivos propuestos por las instituciones gubernamentales y no gubernamentales para hacer frente a esta epidemia, ha sido fomentar el tiempo promedio semanal de actividad física moderada a vigorosa (AFMV) en la población infantil a través de la educación, sobre todo durante la etapa escolar. Cabe destacar que, durante esta etapa, los niños y jóvenes 
se ven enfrentados a cambios físicos, emocionales y psicológicos relacionados con el desarrollo y la maduración, los cuales pueden ser mermados por la obesidad. Además, estos no sólo presentan problemas de salud física (Pedro Delgado-Floody, Caamano-Navarrete, Palomino-Devia, JerezMayorga, \& Martinez-Salazar, 2019), sino que es posible observar depresión (Sutaria, Devakumar, Yasuda, Das, \& Saxena, 2019), baja autoestima (P. Delgado-Floody, CarterThuillier, Jerez-Mayorga, Cofre-Lizama, \& Martinez-Salazar, 2019) y problemas de interacción social (Griffiths, Parsons, \& Hill, 2010), los cuales afectan la motivación para realizar AFMV de forma regular (Martinez-Baena, Mayorga-Vega, \& Viciana, 2018). Todos estos factores no sólo afectan el gasto energético necesario para controlar el peso corporal, sino que también el rendimiento escolar (Marques, Gomez, Martins, Catunda, \& Sarmento, 2017). Ahora bien, estudios previos han señalado que durante la semana, los escolares normopeso y obesos realizan ejercicios de mayor intensidad que los fines de semana (Godard, Roman, del Pilar Rodriguez, Leyton, \& Salazar, 2012; Moreno, Cano, Orellana, \& Kain, 2015). Esto último es relevante, considerando que los niños y jóvenes tienden a compartir más tiempo con sus familias en actividades deportivas a medida que avanza la edad (Viera, Quinones, \& Araujo, 2018), las cuales ocurren en su mayoría los fines de semana. Es reconocido que la clase de educación física (CEF) es la indicada para activar las recomendaciones de ejercicio entregadas por la OMS y arraigar con fuerza los hábitos hacia una alimentación saludable y vida activa durante la adultez. Asimismo, ésta debería ser capaz de motivar a los niños y jóvenes a realizar AFMV regularmente durante toda la semana. En USA, el departamento norteamericano de salud y servicios humanos, recomienda que un niño debería realizar AFMV durante al menos el 50\% del tiempo de la CEF (USDHHS, 2008). En relación al gasto energético, Kahan \& McKenzie (2017) sentenciaron que la CEF puede tener un efecto considerable para el control del sobrepeso y la obesidad de los estudiantes (Kahan \& McKenzie, 2017). Sin embargo, algunos estudios han demostrado tiempos inefectivos de AFMV (RetamalValderrama, Delgado Floody, Espinoza-Silva, \& JerezMayorga, 2018) y limitado gasto energético (da Cunha, Poblacion, Basile, de Aguiar Carrazedo, \& Maia, 2016) durante las CEF. Esto último, fue mayormente observado en niños que poseen valores elevados de IMC (da Cunha et al., 2016; Retamal-Valderrama et al., 2018). Lamentablemente, en la mayoría de estos estudios, la medición de la AFMV fue llevada a cabo con herramientas que tienen algún grado de subjetividad, ya que reúnen información desde la observación directa o cuestionarios. Utilizando acelerómetros, Hortiguela y cols. (2017) observaron un mayor gasto energético en las CEF orientadas a actividades intensas respecto a las menos intensas, alcanzando una de ellas un $\sim 35 \%$ del mínimo gasto energético semanal recomendado en los niños (Hortiguela, Salicetti, \& Hernandez, 2018). Cabe recordar que la intensidad del ejercicio físico y el gasto energético son altamente variables entre los individuos, por lo tanto, se requiere tecnología de alta precisión para evaluarlos (Pasic et al., 2014), aún más si se desea establecer alguna relación de estas variables con la salud (Wong, Day, Luan, Chan, \& Wareham, 2003). El objetivo de este estudio fue evaluar el nivel de intensidad de ejercicio, gasto de energía, número de pasos y actividad metabólica de un grupo de escolares durante una CEF, utilizando acelerometría, y compararlos en relación con la clasificación del estado ponderal medido con el IMC.

\section{Material y métodos}

\section{Participantes}

Escolares ( $\mathrm{n}=128$; 64 niños y 64 niñas) pertenecientes a cuatro instituciones municipales de educación escolar de la ciudad de Quintero (comuna de Quintero, Región de Valparaíso, Chile) fueron seleccionados aleatoriamente desde los niveles 1ro, 2do, 3ro, 4to y 5to básico. Cada nivel tenía dos cursos, de cada uno de ellos fueron reclutados 16 alumnos (ocho niños y ocho niñas). La selección de los alumnos se llevó a cabo sólo en aquellos escolares cuyos padres entregaron previamente su consentimiento para realizar las mediciones. Para esta selección se utilizó la lista de curso separada en niños y niñas, a través de la función de aleatorización del programa Microsoft Excel 2016 (Microsoft Company, United States). El alumno que por razones de salud actual no podía realizar actividad física, fue excluido del estudio y reemplazado por otro de la misma lista. Finalmente fueron excluidos 8 escolares (un niño y siete niñas) que durante la clase presentaron malestar que les impidiera ejecutar el esfuerzo requerido según sus aptitudes normales (dolor músculo-esquelético, esguince, mareo), obteniéndose una muestra final con registros de ejercicio durante la clase de 120 escolares (63 niños y 57 niñas; edad: $8.2 \pm 1.5$ años, peso corporal: $32.9 \pm 9 \mathrm{~kg}$, estatura: $1.32 \pm 0.1 \mathrm{~m})$. En la tabla 1 se observan la edad, características físicas y el estado ponderal de los participantes antes de la acelerometría. El orden de los colegios y la CEF respectiva para las mediciones fueron elegidos aleatoriamente con el mismo programa utilizado para la selección de los participantes. Las 8 clases seleccionadas, una de cada curso, se realizaron en la mañana (8:00-12:00 am) durante la estación de primavera. Para evitar un posible efecto reactivo de los escolares al utilizar un acelerómetro, no se les informó acerca de los objetivos del estudio. Antes de iniciar las evaluaciones, los padres debieron firmar el consentimiento informado escrito. Asimismo, los alumnos y profesores de educación física dieron su asentimiento. Todos los procedimientos fueron llevados a cabo siguiendo las recomendaciones para estudios en humanos descritas en la declaración de Helsinki, y aprobados por el comité científico de ética del Laboratorio de Evaluación de la Condición Física de Quintero (código: 01032015).

\begin{tabular}{|c|c|c|c|c|c|c|c|c|c|c|c|c|}
\hline \multirow{2}{*}{ Variables } & \multicolumn{3}{|c|}{ Todos $(\mathrm{n}=120)$} & \multicolumn{3}{|c|}{ Normopeso $(n=64)$} & \multicolumn{3}{|c|}{ Sobrepeso $(n=24)$} & \multicolumn{3}{|c|}{ Obeso $(n=32)$} \\
\hline & Media & & $\mathrm{DE}$ & Media & & $\mathrm{DE}$ & Media & & $\mathrm{DE}$ & Media & & $\mathrm{DE}$ \\
\hline Edad (años) & 8,2 & \pm & 1,5 & 8 & \pm & 1,5 & 8,4 & \pm & 1,6 & 8,3 & \pm 1 & 1,2 \\
\hline Peso corporal (kg) & 33 & \pm & 9 & 27,1 & \pm & 5,4 & 33,9 & \pm & 7,3 & 42,1 & \pm 7 & 7,4 \\
\hline Estatura (m) & 1,32 & \pm & 0,1 & 1,29 & \pm & 0,09 & 1,33 & \pm & 0,1 & 1,35 & \pm 0 & 0,1 \\
\hline IMC $\left(\mathrm{kg} / \mathrm{m}^{2}\right)$ & 18,8 & \pm & 3,4 & 16,1 & \pm & 1,1 & 18,8 & \pm & 1,3 & 23,1 & \pm 2 & 2,5 \\
\hline IMC z-score & 1,2 & \pm & 1,3 & 0,14 & \pm & 0,54 & 1,3 & \pm & 0,3 & 2,8 & \pm 0 & 0,8 \\
\hline
\end{tabular}

\section{Mediciones}

La medición de la estatura y peso corporal se llevó a cabo en una sala del colegio condicionada (temperatura ambiente entre $20-22^{\circ} \mathrm{C}$ ), utilizando una balanza con 
estadiómetro (modelo BC-520, Tanita Corporation, Tokyo, Japan). Los alumnos debían presentarse con ropa deportiva (la utilizada para la CEF), es decir los niños con pantalón corto y polera, y las niñas con pantalón corto o calzas y polera.

Para observar la actividad metabólica, gasto energético, intensidad y cantidad de ejercicio durante una clase de educación física de 90 minutos orientada al desarrollo de actividad física, se utilizaron acelerómetros triaxiales (modelo GT3X, Actigraph) muy pequeños (3.8 x 3.7 x $1.8 \mathrm{~cm})$ y livianos (27 g). Estos acelerómetros fueron instalados en el costado de la cintura derecha de cada participante. Este dispositivo ha sido previamente validado para medir la aceleración de los movimientos del cuerpo en dirección anteroposterior, vertical y mediolateral (Matthews et al., 2013; Melanson \& Freedson, 1995).

\section{Análisis de los datos}

\section{Estado ponderal}

El estado ponderal fue analizado utilizando el IMC (peso corporal en kg dividido por la estatura en metros elevada al cuadrado). Aquí el programa OMS Anthro (versión 3.2.2, enero 2011) calcula el z-score del IMC por edad según los estándares de crecimiento utilizados por la OMS (de Onis \& Lobstein, 2010). Entonces, los niños fueron clasificados en normopeso (NP) cuando el z-score de IMC fue $\leq 1$ DE $y \geq-1$ $\mathrm{DE}$ (desviación estándar), sobrepeso (SP) cuando fue $>1 \mathrm{DE}$ u obeso (OB) si fue $>2$ DE.

Variables del ejercicio realizado durante la clase de educación física

Las variables del ejercicio fueron cuantificadas luego de digitalizar y filtrar la información contenida en el acelerómetro, con esto se eliminó toda la información que no tuviera características de movimiento humano. El almacenamiento de los registros del acelerómetro se llevó a cabo en epoch de 60 segundos y $30 \mathrm{~Hz}$ de frecuencia de muestreo. La descarga de la información de los acelerómetros se llevó a cabo a través del programa Actilife (versión 6.9.0; ActiGraph LLC, Pensacola, FL). Aquí pudo obtenerse la energía gastada (kilocalorías), el número de pasos ejecutados, el tiempo (minutos) de intensidad de los ejercicios y la actividad metabólica (METs) de cada participante. La ecuación de Trost et al. (1998) fue utilizada para la estimación de los METs (Trost et al., 1998). El gasto energético fue obtenido utilizando el modelo de predicción de regresión lineal de Freedson et al. 1997 (Freedson et al., 1997). En tanto, la intensidad de los ejercicios fueron obtenidos según la recomendación de Freedson et al. (2005) (Freedson, Pober, \& Janz, 2005) para los siguientes puntos de corte para los conteos por minuto (cpm) medidos durante el ejercicio, sedentario: 0-149 cpm, ligero: 150-499 cpm, moderado: 500-3999 cpm, vigoroso: 40007599 cpm, y muy vigoroso >7600 cpm (Freedson et al., 2005). Para la actividad física moderada/vigorosa el criterio seleccionado fue para todo movimiento superior a la intensidad moderada, es decir con un cpm $>500$.

\section{Análisis estadístico}

Los resultados son expresados en promedio y desviación estándar. Se llevó a cabo la prueba de Shapiro-Wilk para observar el tipo de distribución de las variables. La comparación de éstas entre los grupos NP, SP y OB se llevó a cabo con la prueba de one-way ANOVA o Kruskal Wallis según distribución, para comparaciones múltiples se aplicó la prueba de Tukey o Dunn respectivamente. También se observó la asociación entre el IMC y las intensidades de ejercicio realizados durante la CEF con el índice de correlación de Spearman. Para la comparación entre grupos y la asociación de variables se obtuvo diferencias significativas cuando $P$ $<0,05$. Todos los análisis fueron realizados con el programa estadístico Graph Pad Prism 6.01 ${ }^{\circledR}$ para Windows (GraphPad Software Inc., La Jolla, CA, USA).

\section{Resultados}

La prueba de SW determinó que las diferencias promedio para estatura, peso corporal, IMC, gasto energético absoluto (kcal) y la actividad metabólica (MET) fueran analizadas con la prueba ANOVA de una vía (SW: $P>\alpha$ ). Todas las demás variables fueron evaluadas con la prueba de KW (SW: $P<\alpha)$.

En la tabla 2 podemos observar que el gasto energético relativo (kcal/kg) fue menor en los grupos SP y OB respecto a NP con $P<0.05$. Respecto al nivel de ejercicio realizado durante la CEF, la tabla 3 muestra que los OB estuvieron más tiempo en intensidad ligera que NP con $P<0.05$ y menos tiempo de la clase en ejercicio muy vigoroso que el grupo NP con $P<0.05$.
Tabla 2 .

enético, intensidad metabólica y cantidad de movimiento llevada a cabo durante la clase de educación física.

Variables $\quad$ Todos $(\mathrm{n}=120) \quad$ Normopeso $(\mathrm{n}=64)$ Sobrepeso $(\mathrm{n}=24) \quad$ Obeso $(\mathrm{n}=32)$

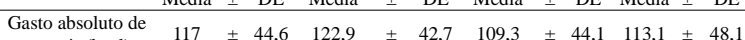

energía (kcal)

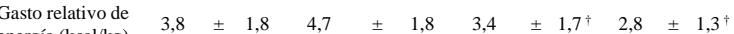

nergía (kcal/kg)

$\begin{array}{lllllllllllll}\text { Actividad } & 3,4 & \pm & 0,7 & 3,5 & \pm & 0,7 & 3,3 & \pm & 0,7 & 3,3 & \pm & 0,8\end{array}$

$\begin{aligned} & \text { Cantidad de } \\ & \text { movimiento }\end{aligned} 2366,7 \pm 629,82315,1 \quad \pm 606,32370,3 \pm 584,22448,6 \pm 710,3$ (número de pasos)

$\mathrm{p}<0,05$ comparado con el grupo normopeso. La significancia estadística para la variable gasto relativo de energía fue medida con la prueba de Kruskal-Wallis, además se aplicó la posprueba Dunn para comparaciones múltiples.

Tabla 3.

Intensidades de esfuerzo llevado a cabo durante una clase de educación física.

Niveles de actividad Todos $(\mathrm{n}=120)$ Normopeso $(\mathrm{n}=64)$ Sobrepeso $(\mathrm{n}=24)$ Obeso $(\mathrm{n}=32$

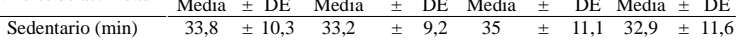
$\begin{array}{lllllllllll}\text { Ligero (min) } \quad 10,1 & \pm 2,5 & 9,5 & \pm & 2,6 & 10,1 & \pm & 2,2 & 11,1 & \pm 2,3\end{array}$

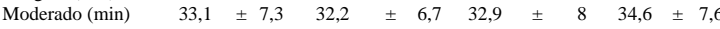
$\begin{array}{llllllllllll}\text { Vigoroso (min) } & 11 & \pm 5,5 & 12,1 & \pm & 6 & 10,3 & \pm & 5,1 & 9,7 & \pm & 4,8\end{array}$

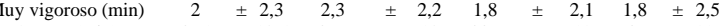
AFMV (min) $\quad 46,1 \quad \pm 10,5 \quad 46,6 \quad \pm \quad 10 \quad 44,9 \quad \pm \quad 11 \quad 46 \quad \pm 11,1$ ${ }^{\dagger} \mathrm{p}<0,05$ comparado con el grupo normopeso. La significancia estadística fue medida con la prueba de Kruskal-Wallis, además se aplicó la posprueba de Dunn para comparaciones multiples.

Por último, en la figura 1 se observa que, a mayor IMC, los escolares tienden a realizar mayor parte del tiempo de la CEF un ejercicio ligero y un menor tiempo de ejercicio vigoroso y muy vigoroso $(P<0.05)$.

\section{Discusión}

Los resultados de este estudio demuestran que los escolares con $\mathrm{OB}$ tienden a gastar menos energía relativa a su peso corporal durante una CEF en relación con NP, realizando la mayor parte tiempo ejercicios de intensidad ligera en desmedro de la intensidad muy vigorosa. Lo anterior se asoció con un menor gasto de energía relativo al peso corporal, 

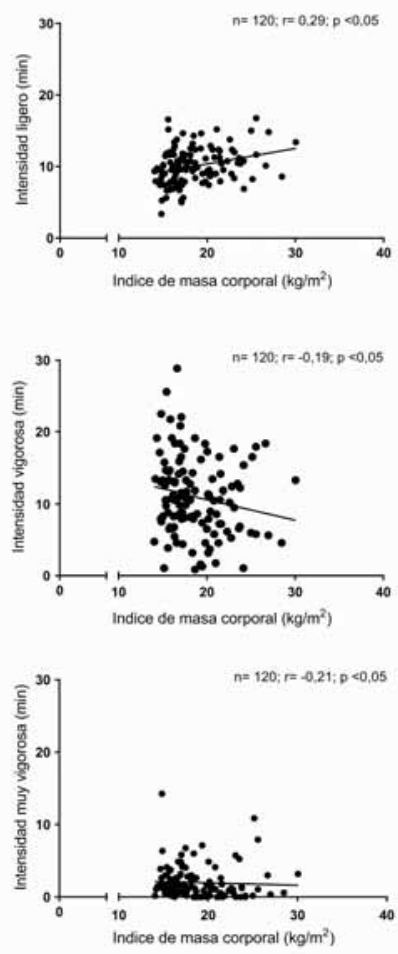

Figura 1. Asociación entre el índice de masa corporal y la intensidad de ejercicio durante una clase de educación física (CEF). r= coeficiente de correlación de Spearman.

sin embargo, el gasto total no presentó diferencias. Además, existió una tendencia en el total de la muestra estudiada a realizar ejercicio físico más ligero, y menos vigoroso y muy vigoroso, en la medida que aumentaba su IMC. Estos efectos pueden estar determinados por un aumento del lastre provocado por un elevado volumen de masa adiposa en el grupo OB.

Previamente, da Cunha et al. (2016) evidenció un mayor gasto energético en escolares cuyos profesores fueron intervenidos con educación teórico-práctica sobre actividad física (estrategias para mantener el movimiento durante la CEF) y nutrición orientados a la salud infantil, respecto al grupo de profesores no intervenidos $(2.54 \mathrm{kcal} / \mathrm{min}$ contra vs $1.81 \mathrm{kcal} / \mathrm{min}$, respectivamente) (da Cunha et al., 2016). En nuestro estudio, los grupos NP, SP y OB obtuvieron promedios de $1.36 \mathrm{kcal} / \mathrm{min}, 1.21 \mathrm{kcal} / \mathrm{min}$ y $1.25 \mathrm{kcal} / \mathrm{min}$ respectivamente, demostrando el bajo gasto energético conseguido durante la CEF. La explicación a esta diferencia puede estar determinada por los menores tiempos de clase registrados por da Cunha et al. (2015), los cuales fueron 27.5 min y 26.2 min para el grupo intervenido y no intervenido respectivamente, a diferencia de los 90 min registrados en nuestro estudio. Un mayor tiempo de clases puede estar relacionado a una menor probabilidad de mantener un ejercicio de intensidad moderada a vigorosa durante toda la CEF, y, por lo tanto, menor gasto energético por minuto. En este punto, es necesario destacar que algunos autores han demostrado que una buena calidad de la CEF permite un gasto energético necesario para el control de peso corporal en los niños, y que incluso, promueve una vida sana hacia la adultez. Esta calidad se centra en mejorar el conocimiento sobre actividad física para la salud y llevar a cabo un seguimiento de metas individuales en tiempo e intensidad del esfuerzo realizado, tanto en profe- sores como alumnos (da Cunha et al., 2016; Kahan \& McKenzie, 2017; Pasic et al., 2014). Para el logro de esto último, la acelerometría demostró que ser fundamental. Apesar que algunos estudios discrepan la validez de la estimación del gasto energético con acelerometría (Alhassan et al., 2012; Nilsson et al., 2008), será necesario considerar el instrumento adecuado de medición, el aspecto del ejercicio que se desea medir y la población evaluada (Crouter, Churilla, \& Bassett, 2006; Troiano, 2009).

Otro resultado relevante de nuestro estudio confirmó que un mayor IMC se asocia con la ejecución de ejercicios de intensidad ligera, perjudicando las intensidades más elevadas (vigorosa y muy vigorosa) durante las CEF. A pesar de lo anterior, es necesario destacar que la AFVM no tuvo diferencia entre los grupos. Cabe recordar que esta variable es utilizada como recomendación para el control del peso corporal con ejercicio físico. En Estados Unidos, el Departamento de Salud y Servicios Humanos recomienda realizar AFMV durante al menos un 50\% del tiempo de la clase de educación física (USDHHS, 2010). En nuestro estudio, el promedio de AFMV de los grupos NP, SP y OB fue 51,7\% (46.6 $\pm 10 \mathrm{~min}$ ), 49,8\% (44.9 $\pm 11 \mathrm{~min})$ y 51,1\% (46 $\pm 11.1 \mathrm{~min})$ respectivamente, los cuales se sobrepasan (NP y OB) o prácticamente alcanzan (SP) los niveles recomendados. Estos resultados son interesantes, ya que normalmente los escolares no están conscientes del nivel de esfuerzo que realizan durante las CEF, a pesar de esto, alcanzaron en promedio los tiempos recomendados de AFMV para el control de la salud. Cabe recordar que los alumnos no fueron informados sobre la función que cumplían los dispositivos, evitando el efecto de voluntariedad para mejorar los resultados. Sin embargo, será necesario que los alumnos utilicen acelerómetros y puedan controlar los avances durante la CEF, de esta forma, estarán conscientes de la frecuencia con que alcanzan sus objetivos de salud. Diferencias de 10 minutos en favor de la AFMV han sido asociados con un mayor control de factores de riesgo cardiometabólicos en niños (Ekelund et al., 2012). Otro parámetro relacionado con la intensidad del ejercicio durante la CEF es la relación profesor/estudiante, la cual ha demostrado ser capaz de subir en 2 min la AFVM en una sesión de ejercicio de 45 min, si el número de alumnos es menor que 25 (Bevans, Fitzpatrick, Sanchez, Riley, \& Forrest, 2010). Estos resultados son alentadores para la población estudiada, sin embargo, habrá que poner atención si estos permiten también alcanzar al menos el objetivo diario. En este contexto, se espera que un niño realice al menos $60 \mathrm{~min} /$ d de AFMV (Alexander, Fusco, \& Frohlich, 2015). Para alcanzar esta meta, en Canadá se creó e implementó un marco de referencia socioecológico conocido como Action Schools! $B C$, cuyos objetivos son entregar oportunidades a los escolares para ser más activos en su contexto escolar, promover la actividad física, dar a conocer sus efectos sobre la salud y facilitar los entornos de apoyo del niño a nivel comunitario, sin alterar el currículo académico (Naylor, Macdonald, Reed, \& McKay, 2006; Naylor, Macdonald, Zebedee, Reed, \& McKay, 2006). Estudios han demostrado que este modelo es efectivo en incrementar la comprensión de los niños respecto a los beneficios de la actividad física (Reed, Warburton, Macdonald, Naylor, \& McKay, 2008) y su práctica (McKay et al., 2015). De esta forma, tomando las recomendaciones de 
Reed et al. (2008), más el uso de acelerómetros para objetivar la AFMV, la población estudiada podría asegurar, al menos durante 2 días a la semana de la jornada escolar en nuestro país, los objetivos requeridos para el control del peso corporal y la salud cardiometabólica.

Otro resultado relevante fue la actividad metabólica registrada, ya que los METs obtenidos demuestran que la intensidad promedio se mantuvo en moderada, pero más cercano al rango de baja (entre 3 y 6 METs) que alta (>6 METs) intensidad(OMS, 2004). Ejercicios realizados a menos METs se relacionan con una menor actividad aeróbica (1 MET= 3.5 $\mathrm{ml} / \mathrm{kg} / \mathrm{min}$ de consumo de oxígeno). De esta forma, un menor gasto energético aeróbico provocará un desmedro en el consumo metabólico principal de ácidos grasos, y, por lo tanto, sobre el control del peso corporal. Junto con esto, pudimos observar que el número de pasos durante la CEF se encuentra muy lejos del objetivo diario deseado, ya que alcanza aproximadamente una quinta parte de lo recomendado (12000) (OMS, 2010a). Si bien, es acosejable incrementar el número de pasos en esta población, creemos que la intensidad vigorosa debería ser el foco principal.

Respecto al efecto del IMC sobre la intensidad de ejercicio, Corder y cols. (2013) observaron que un mayor puntaje $\mathrm{z}$ del IMC (mayor sobrepeso) se asoció con un menor desarrollo de actividades vigorosas en niños entre 10 y 11 años de edad (Corder et al., 2013). Asimismo, O'Connor et al. (2009) demostraron que las intervenciones realizadas por los padres para aumentar el tiempo e intensidad de ejercicio en sus hijos, fuera del ámbito escolar, no han tenido el resultado esperado (O’Connor, Jago, \& Baranowski, 2009). Ahora bien, los colegios son reconocidos como los lugares más confiables para promover las conductas de vida saludables (Pate et al., 2006). En este sentido, es posible que $60 \mathrm{~min}$ de AFMV durante una CEF controlada con acelerómetros, además de una intervención con modelos educacionales similares al Action Schools! BC, favorezcan el aumento del gasto energético a intensidades elevadas de ejercicio dentro y fuera del contexto escolar, pudiendo ser determinante en contrarrestar la aparición de factores de riesgo cardiometabólicos del niño durante su camino a la adultez (Vasquez, Corvalan, Uauy, \& Kain, 2017).

Previamente, Ekelund y cols. (2012) demostraron que la intensidad y duración del del ejercicio en los niños van disminuyendo en la medida que se acercan a la adolescencia (Ekelund et al., 2012). Si bien, este tema no fue investigado en nuestro estudio (limitación del estudio), sí fue posible observar que en el grupo de hombres existió una tendencia a disminuir la intensidad muy vigorosa en la medida que el escolar tenía mayor edad. En un futuro, será necesario investigar los patrones de intensidad de ejercicio durante la CEF a medida que los escolares van creciendo (estudio longitudinal). Además, será necesario diferenciar los resultados según el sexo, ya que estudios han demostrado diferencias en la población infantil (Martinez et al., 2017). Otra limitación de nuestro estudio, fue la baja cantidad de alumnos medidos por nivel, lo cual no nos permitió desarrollar un análisis comparativo según el sexo. .

En conclusión, los escolares NP (entre seis y 11 años) gastan más energía en relación a su peso corporal y a una mayor intensidad de ejercicio que sus pares OB durante una
CEF. Por lo tanto, se recomienda mantener un control de los tiempos e intensidades de AFMV de los escolares durante la CEF para activar los beneficios de salud recomendados, $\mathrm{y}$ así evitar el desarrollo de ECNT en la adultez.

\section{Agradecimientos}

Al Departamento de Administración y Educación Municipal de la Ilustre Municipalidad de Quintero quien favoreció el acercamiento a los colegios y sus comunidades.

\section{Referencias}

Alexander, S. A., Fusco, C., \& Frohlich, K. L. (2015). 'You have to do 60 minutes of physical activity per day ... I saw it on TV': children's constructions of play in the context of Canadian public health discourse of playing for health. Sociol Health Illn, 37(2), 227-240. doi:10.1111/ 1467-9566.12179

Alhassan, S., Lyden, K., Howe, C., Kozey Keadle, S., Nwaokelemeh, O., \& Freedson, P. S. (2012). Accuracy of accelerometer regression models in predicting energy expenditure and METs in children and youth. Pediatr Exerc Sci, 24(4), 519-536.

Bevans, K. B., Fitzpatrick, L. A., Sanchez, B. M., Riley, A. W., \& Forrest, C. (2010). Physical education resources, class management, and student physical activity levels: a structure-process-outcome approach to evaluating physical education effectiveness. J Sch Health, 80(12), 573-580. doi:10.1111/j.1746-1561.2010.00544.x

Blair, S. N. (2009). Physical inactivity: the biggest public health problem of the 21st century. British Journal of Sports Medicine, 43(1), 1-2.

Bouchard, C. (1991). Current understanding of the etiology of obesity: genetic and nongenetic factors. American Journal of Clinical Nutrition, 53(6), S1561-S1565.

Celis-Morales, C., Salas, C., Alvarez, C., Aguilar Farias, N., Ramirez Campillos, R., Leppe, J., . . . Willis, N. (2015). Higher physical activity levels are associated with lower prevalence of cardiovascular risk factors in Chile. Revista Medica De Chile, 143(11), 1435-1443.

Corder, K., Craggs, C., Jones, A. P., Ekelund, U., Griffin, S. J., \& van Sluijs, E. M. (2013). Predictors of change differ for moderate and vigorous intensity physical activity and for weekdays and weekends: a longitudinal analysis. International Journal of Behavioral Nutrition and Physical Activity, 10(1), 69. doi:10.1186/1479-5868-10-69

Crouter, S. E., Churilla, J. R., \& Bassett, D. R., Jr. (2006). Estimating energy expenditure using accelerometers. Eur J Appl Physiol, 98(6), 601-612. doi:10.1007/s00421-0060307-5

Cuadri Fernandez, J., Tornero Quinones, I., Sierra Robles, A., \& Saez Padilla, J. M. (2018). Revision sistematica sobre los estudios de intervencion de actividad fisica para el tratamiento de la obesidad. RETOS. Nuevas Tendencias en Educacion Fisica, Deporte y Recreacion(33), 261266.

da Cunha, C. T., Poblacion, A. P., Basile, F. A., de Aguiar Carrazedo, J. A., \& Maia, M. (2016). Effect of an Educational Program on Schoolchildren's Energy 
Expenditure during Physical Education Classes. MedicalExpress, 3.

de Onis, M., \& Lobstein, T. (2010). Defining obesity risk status in the general childhood population: which cutoffs should we use? International Journal of Pediatric Obesity, 5(6), 458-460. doi:10.3109/17477161003615583

Delgado-Floody, P., Caamano-Navarrete, F., Palomino-Devia, C., Jerez-Mayorga, D., \& Martinez-Salazar, C. (2019). Relationship in obese Chilean schoolchildren between physical fitness, physical activity levels and cardiovascular risk factors. Nutricion Hospitalaria, 36(1), 13-19. doi:10.20960/nh.1932

Delgado-Floody, P., Carter-Thuillier, B., Jerez-Mayorga, D., Cofre-Lizama, A., \& Martinez-Salazar, C. (2019). Relacion entre sobrepeso, obesidad y niveles de autoestima en escolares. RETOS. Nuevas Tendencias en Educacion Fisica, Deporte y Recreacion(35), 67-70.

Ekelund, U., Luan, J., Sherar, L. B., Esliger, D. W., Griew, P., \& Cooper, A. (2012). Moderate to vigorous physical activity and sedentary time and cardiometabolic risk factors in children and adolescents. Journal of the American Medical Association, 307(7), 704-712. doi:10.1001/ jama.2012.156

Freedson, P., Pober, D., \& Janz, K. F. (2005). Calibration of accelerometer output for children. Medicine \& Science in Sports \& Exercise, 37(11 Suppl), S523-530.

Freedson, P., Sirard, J., Debold, E., Pate, R., Dowda, M., Trost, S., \& Sallis, J. (1997). Calibration of the computer science and applications, Inc. accelerometer. Medicine \& Science in Sports \& Exercise, 29(5), 45.

Godard, C., Roman, M., del Pilar Rodriguez, M., Leyton, B., \& Salazar, G. (2012). Variability of physical activity in 4 to $10-$ year-old children: a study by accelerometry. Archivos Argentinos De Pediatria, 110(5), 388-393. doi:10.5546/ aap.2012.388

Griffiths, L. J., Parsons, T. J., \& Hill, A. J. (2010). Self-esteem and quality of life in obese children and adolescents: A systematic review. International Journal of Pediatric Obesity, 5(4), 282-304. doi:10.3109/17477160903473697

Hortiguela, D., Salicetti, A., \& Hernandez, J. (2018). Motivational contrast in physical education depending on caloric expenditure. Revista Internacional De Medicina Y Ciencias De La Actividad Fisica Y Del Deporte, 18(72), 621-634. doi:10.15366/rimcafd2018.72.002

Jebb, S. A., \& Prentice, A. M. (1995). Is obesity an eating disorder? Proceedings of the Nutrition Society, 54(3), 721-728. doi:10.1079/pns19950071

Kahan, D., \& McKenzie, T. L. (2017). Energy expenditure estimates during school physical education: Potential vs. reality? Prev Med, 95, 82-88. doi:10.1016/ j.ypmed.2016.12.008

Marques, A., Gomez, F., Martins, J., Catunda, R., \& Sarmento, H. (2017). Association between physical education, school-based physical activity, and academic performance: a systematic review. RETOS. Nuevas Tendencias en Educacion Fisica, Deporte y Recreacion(31), 316-320.

Martinez-Baena, A., Mayorga-Vega, D., \& Viciana, J. (2018). Predictive factors of physical activity in Spanish students based on their weight status. RETOS. Nuevas Tendencias en Educacion Fisica, Deporte y Recreacion(33),
74-80.

Martinez, C. P., Cuberos, R. C., Sanchez, M. C., Garces, T. E., Ortega, F. Z., \& Cortes, A. P. (2017). Differences between gender in relation with Body Mass Index, diet quality and sedentary activities on children from 10 to 12 year. RETOS. Nuevas Tendencias en Educacion Fisica, Deporte y Recreacion(31), 176-180.

Matthews, C. E., Keadle, S. K., Sampson, J., Lyden, K., Bowles, H. R., Moore, S. C., . . Fowke, J. H. (2013). Validation of a Previous-Day Recall Measure of Active and Sedentary Behaviors. Medicine \& Science in Sports \& Exercise, 45(8), 1629-1638. doi:10.1249/MSS.0b013e3182897690

McKay, H. A., Macdonald, H. M., Nettlefold, L., Masse, L. C., Day, M., \& Naylor, P. J. (2015). Action Schools! BC implementation: from efficacy to effectiveness to scaleup. Br J Sports Med, 49(4), 210-218. doi:10.1136/bjsports2013-093361

Melanson, E. L., \& Freedson, P. S. (1995). Validity of the computer-science and applications, Inc. (CSA) activity monitor. Medicine \& Science in Sports \& Exercise, 27(6), 934-940.

Meneses Montero, M., \& Ruiz Juan, F. (2017). Estudio longitudinal de los comportamientos y el nivel de actividad fisico-deportiva en el tiempo libre en estudiantes de Costa Rica, Mexico y España. RETOS. Nuevas Tendencias en Educacion Fisica, Deporte y Recreacion(31), 219-226.

MINSAL. (2003). Encuesta Nacional de Salud - ENS Chile 2003. Retrieved from http://epi.minsal.cl/wp-content/ uploads/2016/03/presentacion.ens_.vent_.pdf

MINSAL. (2010a). Encuesta Nacional de Salud - ENS Chile 2009-2010. Retrieved from http://epi.minsal.cl/wp-content/ uploads/2016/05/presentacioİ_nENS2010final-20-deenero.pdf.

MINSAL. (2010b). Encuesta Nacional de Salud Chile 20092010. Retrieved from http://epi.minsal.cl/wp-content/ uploads/2016/05/presentacioİ_nENS2010final-20-deenero.pdf.

Moreno, L., Cano, M., Orellana, Y., \& Kain, J. (2015). Compliance of physical activity guidelines by Chilean low-income children; difference between school and weekend days and nutritional status. Nutricion Hospitalaria, 31(5), 2195-2201. doi:10.3305/nh.2015.31.5.8058

Naylor, P. J., Macdonald, H. M., Reed, K. E., \& McKay, H.A. (2006). Action Schools! BC: a socioecological approach to modifying chronic disease risk factors in elementary school children. Prev Chronic Dis, 3(2), A60.

Naylor, P. J., Macdonald, H. M., Zebedee, J. A., Reed, K. E., \& McKay, H. A. (2006). Lessons learned from Action Schools! BC-an 'active school' model to promote physical activity in elementary schools. J Sci Med Sport, 9(5), 413-423. doi:10.1016/j.jsams.2006.06.013

Nilsson, A., Brage, S., Riddoch, C., Anderssen, S. A., Sardinha, L. B., Wedderkopp, N., ... Ekelund, U. (2008). Comparison of equations for predicting energy expenditure from accelerometer counts in children. Scand J Med Sci Sports, 18(5), 643-650. doi:10.1111/j.1600-0838.2007.00694.x

O’Connor, T. M., Jago, R., \& Baranowski, T. (2009). Engaging parents to increase youth physical activity a systematic review. Am J Prev Med, 37(2), 141-149. doi:10.1016/ 
j.amepre.2009.04.020

OECD. (2014). Obesity Update 2014. OECD Heal. Stat. 2014:8. Retrieved from http://www.oecd.org/els/healthsystems/obesity-update.htm

OMS. (2004). Estrategia mundial sobre régimen alimentario, actividad física y salud. Retrieved from https:// ww w. who.int / d i e t p hysicalactivit y/ physical_activity_intensity/es/

OMS. (2010a). Recomendaciones mundiales sobre actividad física para la salud. Retrieved from https://www.who.int/ dietphysicalactivity/factsheet_recommendations/es/

OMS. (2010b). Recomendaciones mundiales sobre actividad física para la salud. . Recuperado el 19 abril 2019. ducir los riesgos y promover una vida sana. Retrieved from https://www.who.int/whr/2002/en/ whr02_es.pdf?ua=1

Pasic, M., Milanovic, I., Janic, S. R., Jurak, G., Soric, M., \& Mirkov, D. M. (2014). Physical activity levels and energy expenditure in urban Serbian adolescents - a preliminary study. Nutricion Hospitalaria, 30(5), 1044-1053. doi:10.3305/nh.2014.30.5.7748

Pate, R. R., Davis, M. G., Robinson, T. N., Stone, E. J., McKenzie, T. L., \& Young, J. C. (2006). Promoting physical activity in children and youth: a leadership role for schools: a scientific statement from the American Heart Association Council on Nutrition, Physical Activity, and Metabolism (Physical Activity Committee) in collaboration with the Councils on Cardiovascular Disease in the Young and Cardiovascular Nursing. Circulation, 114(11), 1214-1224. doi:10.1161/ circulationaha.106.177052

Reed, K. E., Warburton, D. E., Macdonald, H. M., Naylor, P. J., \& McKay, H.A. (2008). Action Schools! BC: a schoolbased physical activity intervention designed to decrease cardiovascular disease risk factors in children. Prev Med, 46(6), 525-531. doi:10.1016/j.ypmed.2008.02.020

Retamal-Valderrama, C., Delgado Floody, P., Espinoza-Silva, M., \& Jerez-Mayorga, D. (2018). Comportamiento del Profesor, Intensidad y Tiempo Efectivo de las Clases de Educación Física en una escuela pública: Un acercamiento a la realidad. RETOS. Nuevas Tendencias en Educacion Fisica, Deporte y Recreacion(35), 4.

Sesso, H. D., Paffenbarger, R. S., \& Lee, I. M. (2000). Physical activity and coronary heart disease in men - The Harvard
OMS. (2012). Informe sobre la salud en el mundo 2002 - Re-

Alumni Health Study. Circulation, 102(9), 975-980. doi:10.1161/01.cir.102.9.975

Singh, A. S., Mulder, C., Twisk, J. W. R., van Mechelen, W., \& Chinapaw, M. J. M. (2008). Tracking of childhood overweight into adulthood: a systematic review of the literature. Obesity Reviews, 9(5), 474-488. doi:10.1111/ j.1467-789X.2008.00475.x

Sutaria, S., Devakumar, D., Yasuda, S. S., Das, S., \& Saxena, S. (2019). Is obesity associated with depression in children? Systematic review and meta-analysis. Archives of Disease in Childhood, 104(1), 64-74. doi:10.1136/archdischild2017-314608

Troiano, R. P. (2009). Can there be a single best measure of reported physical activity? The American journal of clinical nutrition, 89(3), 736-737. doi:10.3945/ ajcn.2008.27461

Troiano, R. P., Berrigan, D., Dodd, K. W., Masse, L. C., Tilert, T., \& McDowell, M. (2008). Physical activity in the United States measured by accelerometer. Medicine and Science in Sports and Exercise, 40(1), 181-188. doi:10.1249/ mss.0b013e31815a51b3

Trost, S. G., Ward, D. S., Moorehead, S. M., Watson, P. D., Riner, W., \& Burke, J. R. (1998). Validity of the computer science and applications (CSA) activity monitor in children. Med Sci Sports Exerc, 30(4), 629-633.

USDHHS. (2008). Physical Activity Guidelines for Americans. Retrieved from https://health.gov/paguidelines/

USDHHS. (2010). Strategies to improve the quality of physical education. Retrieved from http://www.cdc.gov/ HealthyYouth

Vasquez, F. D., Corvalan, C. L., Uauy, R. E., \& Kain, J. A. (2017). Anthropometric indicators as predictors of total body fat and cardiometabolic risk factors in Chilean children at 4, 7 and 10 years of age. Eur J Clin Nutr, 71(4), 536-543. doi:10.1038/ejcn.2016.213

Viera, E. C., Quinones, I. T., \& Araujo, J. A. G. (2018). Relationship between physical activity, nutrition, and family in school-aged youth. RETOS. Nuevas Tendencias en Educacion Fisica, Deporte y Recreacion(34), 85-88.

Wong, M. Y., Day, N. E., Luan, J.A., Chan, K. P., \& Wareham, N. J. (2003). The detection of gene-environment interaction for continuous traits: should we deal with measurement error by bigger studies or better measurement?. International Journal of Epidemiology, 32(1), 51-57. doi:10.1093/ije/dyg002
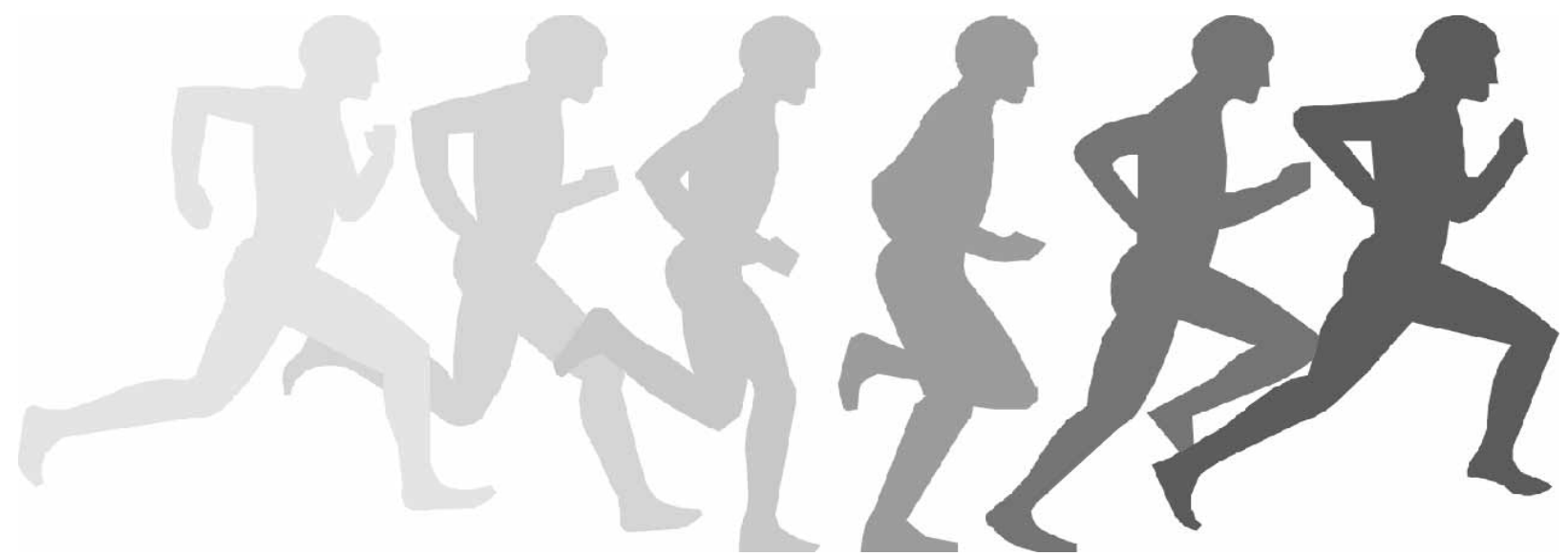